Slavica

bruxellensia

\section{Slavica bruxellensia}

Revue polyphonique de littérature, culture et histoire

slaves

$5 \mid 2010$

Après 1989

\title{
Affronter un lourd passé : débat de presse sur l'Holocauste en Pologne et en Slovaquie après 1989
}

\section{Katrin Van Cant}

Traducteur : Audray Sorio

\section{(2) OpenEdition}

\section{Journals}

Édition électronique

URL : http://journals.openedition.org/slavica/301

DOI : 10.4000/slavica.301

ISSN : 2034-6395

\section{Éditeur}

Université libre de Bruxelles - ULB

Édition imprimée

Pagination : 49-63

ISSN : 2031-7654

\section{Référence électronique}

Katrin Van Cant, «Affronter un lourd passé : débat de presse sur l'Holocauste en Pologne et en

Slovaquie après 1989 », Slavica bruxellensia [En ligne], 5 | 2010, mis en ligne le 15 février 2010, consulté le 04 mai 2019. URL : http://journals.openedition.org/slavica/301 ; DOI : 10.4000/slavica.301

\section{Ce document a été généré automatiquement le 4 mai 2019.}

\section{(c) (i) (9)}

Les contenus de Slavica bruxellensia sont mis à disposition selon les termes de la Licence Creative Commons Attribution - Pas d'Utilisation Commerciale - Pas de Modification 3.0 France. 


\title{
Affronter un lourd passé : débat de presse sur l'Holocauste en Pologne et en Slovaquie après 1989
}

\author{
Katrin Van Cant \\ Traduction : Audray Sorio
}

\section{NOTE DE L'ÉDITEUR}

La langue originelle de cet article est l'anglais

1 Plus de soixante-cinq ans nous séparent des faits et pourtant l'Holocauste est toujours bien présent dans les cercles universitaires et la vie publique ${ }^{1}$. Les implications énormes et la complexité du problème expliquent cet intérêt persistant ainsi que les incessants débats et réinterprétations qu'elle a suscités. Cependant, si le débat sur l'Holocauste dure depuis plusieurs décennies en Europe de l'ouest ${ }^{2}$, il a fallu attendre 1989 pour qu'il soit lancé en Europe centrale. Pendant la période d'après-guerre, le système communiste et son emprise totalitaire sur l'histoire ne laissèrent pas beaucoup de place aux écarts par rapport à la ligne officielle du Parti, qui créa une image déformée des événements de la Deuxième Guerre mondiale, en ce compris le sort des Juifs. Selon le pays et l'époque, la situation des Juifs fut minimisée, détournée ou complètement étouffée.

2 Le présent article examine la façon dont l'Europe centrale aborda la problématique de l'Holocauste après la chute du communisme. Pour cela, il se penchera sur le cas de deux pays, la Pologne et la Slovaquie. Tous deux ont profondément été marqués par la «solution de la question juive » et ont vu une grande partie de leur communauté juive d'avant-guerre périr au cours de la Deuxième Guerre mondiale. Environ 2,9 milions de Juifs polonais et 67000 Juifs slovaques furent assasinés, ce qui correspond à $85 \%$ de la communauté juive polonaise d'avant-guerre et à $75 \%$ de la slovaque ${ }^{3}$. Ces deux pays représentent dès lors d'excellents cas d'étude sur l'interprétation de l'Holocauste après les boulversements politiques de 1989. Cette «page blanche» de leur histoire a-t-elle 
refait surface dès que les deux pays se sont retrouvés sur la voie de la démocratie ? Dans quel contexte ce sujet a-t-il été évoqué, et quel genre de réactions a-t-il suscité ?

Les nombreuses publications ayant pour objet le Vergangenheitsbewältigung ${ }^{4}$ en général et les séquelles de l'Holocauste en particulier présentent une multitude d'approches adoptées envers le passé telles que le silence, les diverses stratégies de négation et "d'oubli », l'attribution de responsabilité et de culpabilité, etc. ${ }^{5}$ Quelles sont celles qui ont été appliquées en Pologne ou en Slovaquie? Ces approches ont-elles évolué et si oui comment expliquer les changements survenus? L'interprétation a-t-elle subi des modifications sous l'influence de facteurs (géo)politiques? La démocratisation a-t-elle introduit une façon plus rationnelle, c'est à dire plus ouverte et plus introspective, d'appréhender l'histoire? L'évolution de l'intégration européenne a-t-elle accéléré le processus de création de récits historiques nationaux?

Ou bien peut-être est-ce le contexte historique spécifique de chaque pays qui a joué un rôle décisif? Après tout, les deux états ont vécu la Deuxième Guerre mondiale et l'expérience communiste qui suivit de façons complètement différentes. La République slovaque a activement collaboré avec l'Allemagne nazie et est sortie largement indemne de la guerre, alors que la Pologne faisait partie des Forces Alliées et a subi des pertes humaines et matérielles extrêmement lourdes ${ }^{6}$. En outre, les deux pays n'ont pas du tout adopté la même attitude envers l'Holocauste. Même si la Pologne n'était pas dépourvue de sentiment anti-juif 7 , la situation n'était pas comparable à celle de la Slovaquie, où l'antisémitisme fut légalement reconnu ${ }^{8}$ et où le gouvernement prit activement part à la déportation de la majorité des Juifs slovaques?.

Quant à l'ère communiste, il a été avancé que le processus de socialisation (en particulier au niveau de l'homogénéisation des interprétations de l'histoire) s'était révélé plus efficace en Slovaquie qu'en Pologne au cours de l'après-guerre ${ }^{10}$. En effet, la Slovaquie, comme beaucoup de pays de l'ancien bloc communiste, s'était modernisée et s'était engagée dans le processus de construction de la nation dans le contexte socialiste. Son expérience nationale faible (et corrompue) et la résistance tout aussi faible que le pays opposait au communisme le rendirent particulièrement vulnérable au processus de socialisation qui lui était imposé. La Pologne en revanche connut une période précommuniste bien plus significative, avec un statut d'état indépendant et des institutions plus solides (comme l'Église de Pologne), au cours de laquelle le passé fut préservé pour résister à la domination communiste.

6 Ces disparités entre les deux pays ont-elles un lien avec la relation au passé qu'ils ont respectivement développée après 1989? La Slovaquie a-t-elle rencontré plus de problèmes pour accepter le passé qui la relie à l'Holocauste, sachant que cela signifiait non seulement affronter les conséquences d'un «oubli délibéré de l'État», mais également assumer la responsabilité de compter parmi les «auteurs»? Ou bien, au contraire, la Pologne a-t-elle eu plus de mal à assumer les accusations de complicité et d'indifférence envers les Juifs, sachant que cela impliquait une remise en question de l'idée de victimisation totale généralement admise dans le pays.

7 Nous tenterons de répondre à ces questions en analysant les débats sur l'Holocauste que l'on retrouve dans la presse des deux pays entre 1989 et 2004. Les sources de presse constituent un média qui a été, et particulièrement ces dernières décennies, d'une importance majeure pour la construction des récits nationaux et qui est encore trop souvent négligé par les spécialistes ${ }^{11}$. Le choix de porter l'analyse sur une longue période de quinze ans permet de retracer les différentes étapes du débat sur l'Holocauste et de 
repérer les points communs et/ou les différences, les continuités et/ou les discontinuités entre les deux pays.

8 L'article commencera par un aperçu des récits communistes d'après-guerre sur l'Holocauste en Pologne et en Slovaquie, en se basant pour ce faire sur de la littérature de seconde main. Il s'intéressera ensuite à la période postcommuniste en s'appuyant cette fois sur des sources de première main, et se poursuivra par une analyse comparative des discours sur l'Holocauste dans deux périodiques de chaque pays : « POLYTIKA » et « WPROST " pour la Pologne, «NOVÉ SLOVo » et « DOMINO EFEKT » pour la Slovaquie ${ }^{12}$. Les sources sélectionnées peuvent toutes être qualifiées de «journaux d'élite » et sont généralement considérées comme représentantes de l'opinion de centre-gauche (« NOVÉ SLOVO » et « POLYTIKA ", qui est parfois aussi simplement qualifié de «centriste ») et de l'opinion de centre-droit (« DOMINO EFEKT » et « WPROST $\left.»^{13}\right)$. Chacun de ces périodiques ont, ou ont eu à un certain moment, une rubrique historique distincte et se sont donc particulièrement intéressés aux sujets d'histoire. En outre, ils ont publié des articles rédigés non seulement par des journalistes, mais également par des historiens professionnels, ce qui permet d'inclure différents niveaux de discours journalistiques.

\section{Partir de zéro ? Récits communistes sur l'Holocauste en Pologne et en Slovaquie ${ }^{14}$}

Il est impossible de comprendre le présent sans connaître le passé. Afin de comprendre les débats de presse sur l'Holocauste dans la Pologne et la Slovaquie postcommunistes, il est dès lors nécessaire de consacrer un peu de temps à la période communiste. Après tout, il serait erroné de croire que le débat sur le sort des Juifs pendant la Deuxième Guerre mondiale est venu de nulle part sur la scène publique après les révolutions de 1989. Il est vrai, par contre, que les autorités communistes ont essayé de monopoliser toutes les interprétations du passé, y compris des événements de la Deuxième Guerre mondiale, qui étaient essentielles aux efforts de légitimation du régime. Bien que certaines sociétés communistes aient été mieux équipées que d'autres pour résister à ces tentatives d'homogénéisation ${ }^{15}$, les discours publics sur l'histoire (y compris dans les organes de presse) étaient aussi efficaces que partout ailleurs à travers le bloc, entièrement dominé par la ligne officielle de l'État.

10 Alors qu'en Pologne la création d'un récit de guerre dogmatique commence directement après la Deuxième Guerre mondiale, le processus débute seulement en 1948 en Tchécoslovaquie, lorsque les communistes prennent le pouvoir. C'est également au cours de ces années-là que l'entreprise d'écriture de l'histoire commence ${ }^{16}$. Les livres et mémoires qui avaient été publiés en Tchécoslovaquie avant 1948 sont alors bannis des rayons des bibliothèques et des librairies.

11 Pour l'essentiel, la version communiste de chaque sujet de guerre ne variait pas et fut, à vrai dire, la même pour tout le bloc socialiste. Il était de l'avis général que le fascisme était mauvais, un produit issu du plus haut degré du capitalisme, même si d'ordinaire on ne donnait pas d'explication sur ce qu'il avait de mauvais. La résistance au fascisme était héroïque et menée par les communistes, en particulier par l'Union Soviétique. L'importance de l'Holocauste était minimisée et le sort des Juifs en tant que victimes particulières n'était pas spécialement mis en avant. 
12 Toutefois, ces sujets furent abordés différemment en Slovaquie et en Pologne, et les approches évoluèrent avec le temps. Lorsqu'il s'agissait des relations entre Juifs et Polonais pendant la Deuxième Guerre mondiale, le récit polonais officiel insistait sur la souffrance partagée par les Polonais et les Juifs et sur l'aide polonaise qui s'était étendue aux Juifs. Les historiens décrivirent l'aide apportée aux Juifs par les Polonais ainsi que leur passivité suite aux représailles nazies, comme la réponse principale des Polonais au génocide nazi perpétré sur leur sol ${ }^{17}$. Le censeur du gouvernement n'autorisait que les publications mettant l'accent sur cette aide apportée aux Juifs ; travaux qui concordaient avec le récit officiel sur l'héroïsme et la résistance des Polonais en temps de guerre ${ }^{18}$. Ce récit empreint de dénégation continua à façonner la mémoire collective polonaise de la guerre pendant toute la période communiste.

13 Suite à plusieurs événements au cours des années 1980, un vent de réconciliation et de franchise souffla sur les cercles universitaires, et l'idée que se faisaient les Polonais des Juifs, du judaïsme et de la culture juive changea ${ }^{19}$. Des études virent le jour, qui, pour la première fois, soumettaient les hypothèses communément acceptées sur le comportement de la Pologne durant la guerre à une enquête académique impartiale ${ }^{20}$. La première contestation publique au récit polonais prédominant sur l'héroïsme et le martyre suivit en 1987, lorsque le professeur Jan Błoński publia un article intitulé « Les pauvres Polonais regardent le ghetto " dans l'hebdomadaire catholique à tendance libérale «TYGODNIK POWSZECHNY ${ }^{21}$. Pour la première fois, l'article brisait résolument le silence officiel. Błoński dénonça non seulement l'attitude générale des Polonais envers les Juifs persécutés dans la Pologne en guerre, mais également celle des Polonais contemporains face à ce problème. Sans surprise, cet article déclencha un débat à l'échelle nationale sur les relations judéo-polonaises à l'époque de la guerre. Bien que l'article fut critiqué et l'est encore à ce jour par quelques historiens polonais pour son manque de preuve et l'abondance d'hyperboles ${ }^{22}$, sa parution est largement reconnue comme un moment-clé du débat judéo-polonais sur la Deuxième Guerre mondiale.

semble un peu plus compliquée en Slovaquie, puisque l'atomisation au sein du Parti communiste (reflétée par les fréquentes épurations) et les déformations de l'histoire durèrent pendant plus de dix ans, bien plus longtemps donc que dans la plupart des autres pays du bloc de l'Est. Au cours des années 1948-1961, les versions officielles de l'histoire changeaient rapidement et étaient incohérentes, à tel point qu'il y avait peu de chance que l'une d'entre elles soit majoritairement adoptée. Dans le récit officiel de la Deuxième Guerre mondiale (par exemple dans les manuels des années 1950²3), les Juifs slovaques étaient souvent tout simplement oubliés. S'ils étaient déjà mentionnés dans le contexte des déportations slovaques, leur place spécifique en tant que victimes n'était pas reconnue. Aucun chiffre n'était cité et il ne figurait nulle part que l'envoi des Juifs dans les camps faisait partie d'une tentative nazie d'extermination à l'échelle européenne. Les communistes étaient systématiquement décrits comme les victimes principales du fascisme. Jozef Tiso, le prêtre catholique président de la République slovaque pendant la Deuxième Guerre mondiale, exécuté en 1947, était présenté comme le plus grand méchant de la République slovaque (fasciste) de l'époque de la guerre.

Les années 1960, une période de réformes au sein du Parti communiste tchécoslovaque, virent paraître une longue série de publications qui s'intéressaient particulièrement aux déportations juives et effleura parfois même la question de la responsabilité (slovaque) ${ }^{24}$. Entre 1948 et 1989, ce n'est qu'au cours de ces quelques années qu'une telle discussion eut lieu. Sur le plan officiel, par contre, le chapitre juif était toujours minimisé ${ }^{25}$, mais cette 
fois, d'autres raisons motivaient le silence à ce sujet. Les communistes slovaques, mus par le désir nationaliste de se trouver un passé approprié, évitèrent d'assumer officiellement la responsabilité des déportations, comme cela avait été le cas dans les pays d'Europe de l'ouest. Cette attitude contrastait clairement avec celle des années 1950, lorsque l'oubli organisé et l'atomisation de l'élite étaient à la base de la fuite de responsabilité. Le chapitre juif avait une priorité moindre par rapport à la demande slovaque d'autonomie ${ }^{26}$ et il n'y avait que peu de temps à lui consacrer. Après 1969 (et la brèche du Printemps de Prague), l'affaire s'estompa dans la vague d'antisémitisme officielle qui suivit la Guerre des Six jours au Moyen Orient en 1967.

Les deux décennies qui suivirent, généralement mentionnées dans l'historiographie comme la période de normalisation, marquèrent le retour à un oubli délibéré de l'État ${ }^{27}$. Les travaux critiques publiés au cours des années 1960 furent retirés de la vente et disparurent des bibliothèques. L'élimination affecta directement les écrits historiques, puisque les historiens détracteurs perdirent leur position ou ne furent plus en mesure de publier. Les discussions historiques continuèrent uniquement en samizdat. En accord avec la transformation officielle du récit, les informations sur le problème juif furent de nouveau réduites à peau de chagrin et privées de leur contexte ${ }^{28}$.

En conclusion, nous pouvons constater que, vers la fin des années 1980, les deux pays se trouvaient dans une phase fondamentalement différente du débat sur l'Holocauste. Alors qu'en Slovaquie de nouvelles tentatives pour intégrer les Juifs dans le récit officiel de guerre semblaient encore lointaines, la Pologne, elle, semblait avoir pris son élan en la matière.

\section{9 et ses répercutions dans le débat sur I'Holocauste en Pologne et en Slovaquie}

Le climat de liberté qui règne en Pologne et en Tchécoslovaquie après les révolutions de 1989 ouvre la voie à une renaissance partielle de la vie juive et à une présence plus marquée sur la scène publique des sujets liés aux Juifs. Dès Noël 1990, le Parlement slovaque adopte une résolution exprimant un profond regret pour la déportation des Juifs slovaques. Du côté polonais, le président Lech WałĘsa pose un geste d'envergure le 21 mai 1991, avec son discours devant le Parlement israélien, au cours duquel il demande publiquement pardon à la communauté juive ${ }^{29}$. Pologne et Slovaquie commencent à travailler sur les lois de restitution des biens juifs spoliés pendant la guerre, bien que celles-ci ne rencontrent qu'un succès relatif ${ }^{30}$. Des livres et des films sur le destin des Juifs pendant la Deuxième Guerre mondiale font leur apparition dans les sphères académique et culturelle, et le processus de commémoration s'amorce dans les espaces publics ${ }^{31}$. Ces changements favorables se frayent un chemin jusque dans la presse, même si la couverture des sujets associés aux Juifs par la presse slovaque de l'époque reste très limitée, tant au niveau de la qualité que de la quantité. Pendant qu'en Pologne des affaires sensibles comme celle du Carmel d'Auschwitz (voir plus loin) font déjà objet de débats, la presse slovaque se cantonne à des sujets tels que la restitution des biens ou la dernière controverse suscitée par le livre de Daniel Goldhagen, Hitler's Willing Executioners (1996) ${ }^{32}$. Ces modestes tentatives laissent présager un futur prometteur pour le débat sur l'Holocauste dans les deux pays. Et pourtant leur contexte sociopolitique respectif ainsi que les tabous et/ou les "pages blanches » hantant les deux états se révéleront être de sérieux obstacles sur la route du jugement ouvert et critique sur le passé de la nation. 
19 En 1993, la scission de la Tchécoslovaquie et la constitution de la République slovaque sont source de nouveaux défis pour la Slovaquie. Outre les problèmes sociopolitiques issus de la révolution de 1989, le pays doit désormais également faire face aux problèmes de légitimation et au besoin encore plus urgent de renforcer son identité nationale par le biais d'un récit historique cohérent ${ }^{33}$. Avec cette quête de reconnaissance et de continuité, c'est naturellement la République slovaque de guerre qui fait surface, puisqu'elle constitue la seule (et pas la moindre) expérience d'État « indépendant » dans l'histoire de la Slovaquie ${ }^{34}$. En réalité, les discussions sur le sujet font déjà rage depuis le début des années 1990 au sein des élites slovaques dirigeantes ${ }^{35}$, mais ce n'est qu'à partir de 1993 qu'elles revêtissent une importance majeure dans la presse. Fait assez remarquable et hautement emblématique, les Juifs furent exclus du débat.

20 La multitude d'articles publiés peut être grossièrement répartie en deux groupes. Dans le premier, des écrits sur la République slovaque dans un contexte de légitimation et de continuité, ainsi que de longues analyses sur la nature du premier État slovaque et sur le contexte dans lequel celui-ci est né36. La République slovaque de l'époque de la guerre y est lourdement critiquée pour avoir été un État vassal d'Hitler plutôt qu'un État souverain et le Soulèvement national slovaque est présenté comme la seule éclaircie de cette période sombre. Dans ces circonstances, le Parlement de la République slovaque actuelle est considéré comme le successeur légitime du Conseil national slovaque établi après le Soulèvement national ${ }^{37}$.

21 Le second groupe d'articles, qui est aussi le plus fourni, étudie et en général critique les efforts de réhabilitation de la République slovaque de l'époque de la guerre et de son président Tiso ${ }^{38}$, qui ont pu être observés à l'époque dans la société slovaque. Ces tentatives sont à prendre en compte dans le cadre du mandat de Vladimir Mečiar et de ses partenaires de coalition nationalistes en Slovaquie, qui (défendaient et) prônaient activement le retour à cet épisode d'indépendance slovaque ${ }^{39}$.

22 Alors que le premier groupe d'articles provient presque exclusivement de la revue de centre-gauche (« NOVÉ SLOVO BEZ REŠPEKTU »), ceux du second groupe se retrouvent tant dans «NOVÉ SLOVo" que dans " DOMINO EFEKT». L'idéologie politique de ces deux périodiques peut expliquer ce fait. Tous deux ont adopté une attitude critique envers le gouvernement de Mečiar, et "NOVÉ SLOVo » était réputé pour son soutien au parti des anciens communistes $\left(\mathrm{SDL}^{40}\right)$, qui considérait le Soulèvement national slovaque comme l'une des pierres angulaires de la conscience nationale slovaque ${ }^{41}$.

23 Les Juifs ne figurent dans aucun des deux groupes d'articles ou sont simplement mentionnés en termes voilés. Lorsque la réhabilitation de Tiso et de son État y est critiquée, les accusations se dirigent contre "la propagation du fascisme ", mais les arguments pour appuyer ces accusations sont rares. Les articles ont tendance à adopter un ton très passionné. En d'autres termes, et pour résumer, on pourrait qualifier le débat sur l'Holocauste au cours de la première moitié des années 1990 en Slovaquie, de "décollage raté». Bien que la discussion émergeante sur la République slovaque de guerre ait offert de nombreuses opportunités, l'Holocauste (c'est à dire le destin des Juifs slovaques durant la Deuxième Guerre mondiale) n'a jamais été inclus dans le débat. Le problème juif n'apparaissait qu'indirectement (dans les réactions aux tentatives de réhabilitation de la République de Tiso) et lorsque c'était le cas, il était évoqué de façon superficielle et sans aucune distance émotionnelle. Les articles ont tendance à distinguer l'État slovaque et le régime qui le gouverne ${ }^{42}$, et à dissocier Tiso de sa position antisémite. 
Probablement une des conséquences du désir d'envisager l'État comme quelque chose de positif - sans tenir compte des circonstances dans lesquelles il s'est érigé.

En contraste au «décollage raté » de la Slovaquie, le débat sur l'Holocauste est lancé directement après 1989 en Pologne, même si ce fut principalement de façon indirecte. La controverse du Carmel d'Auschwitz, qui fit rage en Pologne pendant toute l'année 1989 et le début de $1990^{43}$, reflète très bien cette situation. Bien que le conflit date déjà de 1984, puisqu'à l'époque l'ouverture du couvent avait déclenché des protestations dans le monde entier, il atteignit son point culminant en 1989, lors d'une tentative des Juifs américains pour entrer de force dans le bâtiment ${ }^{44}$. Des gens issus de toutes les couches de la population (historiens, journalistes, simples lecteurs, juristes) participèrent au débat et le point de vue des Polonais fut aussi bien représenté dans la presse que celui des Juifs.

La mémoire de l'Holocauste et la question de déterminer qui, des Juifs ou des chrétiens, pouvait revendiquer cette mémoire se retrouvèrent pris dans la controverse. Il s'agissait plus précisément d'une querelle portant sur Auschwitz en tant que symbole principal de la souffrance de la Deuxième Guerre mondiale, et de la compétition entre Juifs et Polonais pour s'arroger le statut de victime ultime. Les Juifs furent accusés d'essayer de "judaïser» la mémoire d'Auschwitz, réduisant la mémoire de la Deuxième Guerre mondiale à la Shoah et tous les crimes perpétrés à Auschwitz, à l'extermination des Juifs. Les Polonais, quant à eux, furent accusés d'être antisémites et d'essayer de " christianiser » la mémoire d'Auschwitz, au mépris de la sensibilité juive. Finalement, à la demande du pape, les carmélites du couvent acceptèrent de déménager ${ }^{45}$ et l'affaire fut enterrée, avant de refaire surface à la fin des années $1990^{46}$. Cependant, elle ne fut plus en mesure de provoquer le genre de réactions qu'elle avait suscité en 1989.

$\mathrm{Au}$ cours des années qui suivirent (milieu des années 1990), l'affaire du Carmel d'Auschwitz donna le ton aux débats judéo-polonais qui continuèrent à être extrêmement passionnés et se concentrèrent sur le statut de victime des Polonais. Les articles sur les Juifs (polonais) qui périrent dans les camps de concentrations sont représentatiff ${ }^{47}$. La question du statut de vétéran pour les Polonais qui avaient sauvé la vie de leurs compatriotes juifs durant la guerre fit brièvement surface et plaça cette fois les Polonais dans le camp des héros (le deuxième facteur dominant dans le récit polonais de la Deuxième Guerre mondiale) ${ }^{48}$. Parallèlement à cela, des signes positifs semblaient pourtant indiquer que le débat polonais s'ouvrait à la critique. À cet égard, mentionnons par exemple que l'antisémitisme polonais en tant que phénomène historique figurait constamment dans les articles de presse relatifs aux Juifs, comme ce fut le cas pendant l'affaire du Carmel. Cela fut en conséquence sévèrement critiqué ou tourné en ridicule dans des bandes dessinées en raison du son caractère irrationnel ${ }^{49}$. Cependant, l'antisémitisme polonais n'était pas encore traité comme un sujet à part entière ${ }^{50}$, et n'était presque jamais associé à la Deuxième Guerre mondiale ${ }^{51}$. La compétition entre Juifs et Polonais pour obtenir le statut de victime et de martyre continuait ${ }^{52}$.

\section{Élargir le débat. Un pas en avant?}

27 À partir de 1995, le débat connaît une ouverture en Slovaquie et en Pologne. Alors qu'en Pologne les commémorations du pogrom de Kielce font de l'antisémitisme polonais un sujet à part entière et mettent les Polonais face à leur image (non seulement de victimes, mais aussi de coupables), en Slovaquie, le problème juif arrive progressivement sur le devant de la scène. 

guerre, ce qui signifie que l'Holocauste des Juifs slovaques est enfin reconnu comme une part intrinsèque de cette période d'indépendance slovaque ${ }^{53}$. On continue pourtant à négliger les déportations slovaques et celles-ci interviennent toujours lorsque le fond de la discussion porte sur l'État slovaque lui-même. La plupart du temps, les déportations sont condamnées de façon univoque, mais la question de la culpabilité est souvent mise de côté. Et lorsque cette question a déjà été posée, la tendance générale est d'adopter une attitude de "négation détournée » : la faute étant principalement à rejeter sur un petit groupe de personnalités radicales comme le Premier ministre Vojtech Tuka et le Commandant en chef de la Garde de Hlinka, Alexander $\mathrm{Mach}^{54}$. La responsabilité politique de Tiso est reconnue, mais on prétend également qu'il n'eut guère d'autres choix à l'époque.

Dans un deuxième temps, la politique anti-juive de l'État slovaque de l'époque de la guerre devient le sujet principal des articles et fait pour la première fois l'objet d'une analyse plus détaillée. La qualité de ces articles varie cependant considérablement au niveau de la profondeur, de l'utilisation appropriée des sources et de l'espace laissé à la discussion. La récurrence de certains arguments et l'insistance sur certains événements considérés comme des tournants dans la mise à exécution de l'Endlösung des Juifs slovaques sont caractéristiques.

En Pologne, le $50^{\mathrm{e}}$ anniversaire des événements survenus à Kielce introduisit la question de la culpabilité dans le débat sur l'Holocauste ${ }^{55}$. Néanmoins, les faits en cause s'étant déroulés après la guerre et étant de surcroît éclipsés par la théorie d'un acte de provocation communiste (théorie qui ne fut jamais prouvée ni démentie ${ }^{56}$ ) l'image que les Polonais avaient d'eux-mêmes et leur attitude de victimisation totale fut momentanément préservée. La vraie mise à l'épreuve eut lieu en 2000, lorsque l'affaire Jedwabne explosa. Puisque cette affaire constitue un passage clé dans le débat sur l'Holocauste en Pologne, les paragraphes suivants lui seront spécialement consacrés. En ce qui concerne la Slovaquie, nous analyserons plus en détail une série d'articles (faisant partie du «deuxième temps » introduit plus haut) consacrés aux politiques juives de la République de guerre, puisqu'eux aussi représentèrent un certain progrès dans le débat sur l'Holocauste en Slovaquie. Les Polonais tout autant que les Slovaques durent s'interroger sur la notion de culpabilité et de complicité (personnelle ou collective).

\section{Défier les limites - L'affaire Jedwabne}

31 En mai 2000, Jan Tomasz Gross publie un livre, SĄsiedzi: Historia zagłady żydowskiego miasteczka ${ }^{57}$, dans lequel il décrit le massacre de 1600 Juifs par leurs voisins polonais dans la ville de Jedwabne, en juillet 1941. Bien que le livre de Gross ne soit pas la première publication sur le massacre de Jedwabne et qu'il n'ait pas fait l'objet d'un grand tirage, il a déclenché un immense débat en Pologne. La raison de ce tumulte tient dans le fait que les Polonais se retrouvèrent confrontés sans ambages à ce que Błoński qualifiait déjà en 1987 de «plus grande crainte liée aux relations judéo-polonaises pendant la Deuxième Guerre mondiale », autrement dit la peur d'être assimilé aux «assistants de la mort » ${ }^{58}$, la peur d'être accusé d'avoir été complices de la Solution finale. Dans cet article, Błoński décrivait également le comportement type auquel ses compatriotes avaient d'ordinaire recours lorsqu'ils se trouvaient confrontés à de telles allégations : non seulement ils évitaient farouchement d'aborder le sujet (typique des années 1990), mais ils donnaient également 
toutes sortes de justifications et ressortaient des stéréotypes sur les Juifs pour insister sur le fait qu'ils n'étaient pas irréprochables.

Plus de dix ans plus tard, cette description était plus que jamais d'actualité. Tout le stock de stéréotypes polonais sur les Juifs s'infiltra dans la presse qui couvrait le débat de Jedwabne, mais plutôt que de servir de justifications, ils furent lourdement critiqués ${ }^{59}$ et qualifiés de réactions immatures de la part de la société polonaise (sauf dans la presse d'extrême droite ${ }^{60}$ ). Le stéréotype żydokomuna, également appelé «l'argument du double génocide $»^{61}$ dans la littérature contemporaine, fut par exemple souvent mis en cause. Cet argument consistait à minimiser et à excuser les erreurs du passé afin de justifier jusqu'à un certain point les crimes contre les Juifs. Pour ce faire, le judaïsme était assimilé au communisme et le rôle joué par les Juifs communistes dans l'instauration de la domination communiste en Pologne était exagérément amplifié.

L'avis général adopté par la presse polonaise était positif dans le sens où il brisait de nombreux tabous et posait un regard critique non seulement sur les événements de Jedwabne de 1941, mais également sur la façon dont la société polonaise avait traité ce qui était décrit comme une grande "tragédie $»^{62}$ et un grand "poids » ${ }^{63}$ sur la conscience polonaise. Plus d'une fois, les Polonais furent invités à ressentir une " honte collective", puisqu'ils n'avaient jamais eu de mal à ressentir une "fierté collective " pour les réalisations individuelles des personnages historiques polonais ${ }^{64}$.

34 Cependant, d'un point de vue linguistique, la critique acerbe formulée dans ces articles faisait parfois plutôt preuve de sensationnalisme et ne contribuait dès lors pas vraiment à nuancer la vision sur le sujet. Citons en exemple des titres (accrocheurs) comme « Mord rytualny" (Un Meurtre rituel) ou "Całopalenie " ${ }^{65}$ (Brûlés vifs) et l'évocation des massacres cosaques ("rzezie kozackie ${ }^{66}$ ) de la Pologne des XVI et XVII ${ }^{e}$ siècles pour qualifier les événements de Jedwabne. Outre cela, la communauté moderne de Jedwabne était unilatéralement dépeinte de façon négative. Les habitants étaient des incarnations de "l'Homo jedvabicus», atteints du "syndrome de Jedwabne». Ces deux expressions étaient utilisées pour cataloguer les quartiers polonais qui étaient restés antisémites et intolérants ${ }^{67}$. Ce procédé permettait de rejeter la responsabilité des meurtres de Jedwabne sur une poignée " d'étrangers », des membres atypiques de la nation polonaise, et n'était pas sans rappeler la "négation détournée » vue plus haut lorsque nous avons évoqué la mémoire slovaque de l'Holocauste.

Comme les paragraphes précédents le montrent, la controverse de Jedwabne fut d'une importance cruciale puisqu'elle bouleversa la conscience de la nation et intégra les affaires les plus polémiques de la Deuxième Guerre mondiale au débat polonais sur l'holocauste. Cette affaire eut, par ailleurs, un certain retentissement dans la sphère académique puisqu'on procéda à un important archivage ${ }^{68}$ et à une réévaluation de ces faits historiques, par le biais d'une enquête menée par l'Institut de la mémoire nationale (Instytut pamięci Narodowej, IPN) en 2000-2001. Un important groupe d'intellectuels polonais cautionna les découvertes de Gross et commença à débattre de celles-ci dans des articles, essais, revues, programmes télévisés et programmes radiophoniques. Au niveau politique, le Président, le Premier ministre et une centaine d'évêques environ présentèrent des excuses publiques. Au cours de la commémoration du $60^{\mathrm{e}}$ anniversaire du massacre, un nouveau monument fut érigé en hommage aux victimes de Jedwabne.

Toutefois le débat montra aussi clairement ses limites. Celles-ci se manifestèrent premièrement dans la discorde qui anima le pays concernant les excuses, la cérémonie 
commémorative, et jusqu'aux inscriptions du monument ${ }^{69}$. Deuxièmement, le fait que les débats de presse, même les plus critiques (à l'exception de la gamme nationaliste d'extrême droite), éprouvent de grosses difficultés à rester émotionnellement neutres, et soient tentés de diaboliser un groupe choisi (et ainsi acquitter la majorité), illustre certains des problèmes récurrents. Enfin, en omettant de relier le cas de Jedwabne à des actes similaires d'agression contre les Juifs au cours de la guerre (comme le pogrom de Radziłow le 7 juillet $1941^{70}$ ), la presse ne parvint pas à placer Jedwabne dans un contexte plus large et ainsi à permettre aux Polonais de passer outre leur lourd passé.

Après 2001, la controverse de Jedwabne a progressivement disparu de la presse, bien qu'elle ait fermement secoué le monde universitaire. Au cours des années qui suivirent, les articles traitant de la question judéo-polonaise exprimèrent principalement des sentiments d'espoir pour le futur de ces relations du passé entre Juifs et Polonais. On continua à promouvoir l'image (qui était loin d'avoir disparu) des Polonais victimes de la Deuxième Guerre mondiale, et le discours national du «héros » connut une nouvelle jeunesse. Lentement mais sûrement, les Juifs ont fini par être associés à la résistance de la Deuxième Guerre mondiale ${ }^{71}$. En d'autres termes, ils n'étaient plus seulement décrits comme des victimes passives ayant été, tels des agneaux, à l'abattoir.

\section{L'Holocauste en Slovaquie}

Vers la fin des années 1990, les limites du débat sur l'Holocauste en Slovaquie furent considérablement repoussées lorsque les articles de presse traitant de l'histoire nationale se concentrèrent sur les politiques anti-juives de la République slovaque de l'époque de la guerre. Pourtant le contenu de ces articles divergeait au niveau de la valeur, laissant entrevoir que le débat sur la Deuxième Guerre mondiale en Slovaquie ne mûrirait jamais.

Alors que la plupart des articles se caractérisaient par une vaste documentation basée sur les faits (un important contraste avec le peu d'attention que le sujet avait rencontré au cours des années précédentes), la neutralité avec laquelle ces faits étaient présentés laissait encore beaucoup à désirer. Le manque de distance et le ton passionné employé étaient les aspects les plus problématiques. Bien que cela ne les ait pas empêchés de briser le silence qui entourait toujours le "problème juif », beaucoup d'entre eux ne réussirent pas à rester objectifs dans leur propos et, de ce fait, perdirent beaucoup en crédibilité. Énumérations de chiffres sans les placer dans un contexte approprié, manque de transparence dans l'utilisation des sources et implication émotionnelle de l'auteur constituent de bons exemples ${ }^{72}$.

Cependant on publia de plus en plus d'articles écrits par des historiens professionnels, ce qui se répercuta la plupart du temps dans l'attitude critique adoptée envers le régime de la République slovaque de l'époque de la guerre. La responsabilité politique de Tiso ne fut plus longtemps mise en doute, et le président slovaque fut parfois mis sur le même pied que Tuka et Mach $^{73}$ (qui dans d'autres articles étaient toujours considérés comme des « extrémistes »). Néanmoins, la figure de ce prêtre catholique semblait toujours être à l'épreuve des attaques sérieuses ${ }^{74}$. Deux événements sont généralement considérés comme des tournants dans le cadre des déportations slovaques: la Conférence de Salzbourg en juillet 1940, suite à laquelle le processus de nazification commença en Slovaquie, et l'introduction du Codex juif en septembre 194175. Ce dernier (souvent comparé aux Lois de Nuremberg), en particulier, reçut beaucoup d'attention puisque de nombreux articles se consacrèrent entièrement à l'analyse des différentes étapes qui 
menèrent à l'adoption officielle du Codex par l'État slovaque. On mit l'accent sur la singularité de cette série de législations anti-juives dans le contexte de l'histoire de l'Europe moderne.

41 Ces analyses des politiques antisémites de la République slovaque de l'époque de la guerre ne se penchèrent pas suffisamment sur la question de la collaboration en dehors du régime. À cet égard l'attitude de l'Église catholique slovaque envers le régime fasciste était de plus en plus critiquée ${ }^{76}$ mais le rôle du Slovaque moyen en tant que complice de l'Holocauste restait un énorme point d'interrogation. Au lieu de cela, le nombre relativement important d'articles traitant de l'horreur des camps de concentration soulignait systématiquement la nature inhumaine du régime nazi ${ }^{77}$.

\section{Conclusion}

De grandes différences mais aussi des points communs frappants dans l'approche et l'attitude adoptées face au lourd passé de la Deuxième Guerre mondiale ressortent de cette analyse des débats de presse sur l'Holocauste en Pologne et en Slovaquie après 1989. À la lumière du passé, des facteurs externes semblent avoir été à l'origine de ces différences, le facteur le plus important étant de nature historique et socioculturelle.

Le débat sur l'Holocauste, issu de divergences sociopolitiques et de l'élaboration des récits de la Deuxième Guerre mondiale pendant l'ère communiste, connut un point de départ fondamentalement différent en Pologne et en Slovaquie. Tandis qu'en Slovaquie la période de normalisation étouffait les tentatives de réforme et écartait presque complètement les Juifs du discours public, en Pologne, l'amélioration des relations entre Juifs et Polonais au cours des années 1980 conduisait à une contestation publique du récit officiel (l'article de Błoński).

Les révolutions de 1989 eurent des conséquences immédiates sur le débat sur l'Holocauste dans la presse polonaise, alors qu'en Slovaquie ces conséquences furent marginales. Résultat: la presse polonaise publia un grand nombre d'articles sur des sujets controversés liés aux Juifs alors que la presse slovaque se limita à un petit nombre d'articles sur des sujets qui ne risquaient pas de compromettre l'image de la nation. Au début des années 1990, le contenu du débat sur l'Holocauste en Slovaquie et en Pologne présenta des différences que l'expérience spécifique de la Deuxième Guerre mondiale de chaque pays, entre autres, pouvait expliquer. Dans le cas de la Pologne, la guerre avait profondément entaillé les relations entre Juifs et Polonais et le débat s'était installé dans un climat hostile, dominé par la course au statut de victime absolue. Tandis qu'en Slovaquie, les relations entre Juifs et Slovaques pendant la guerre constituaient des pages noires de l'histoire nationale et étaient fermement écartées. Même lorsque, et plus particulièrement en 1993, le processus de construction de la nation commença et que les hommes politiques slovaques se mirent à « écrire l'histoire de la République de guerre ", aucun débat sur l'Holocauste ne fut introduit.

La suite du débat sur l'Holocauste dans les deux pays peut être décrite comme un processus d'intégration de ce lourd passé dans le récit national. Tandis qu'en Slovaquie on part d'une situation où l'Holocauste est totalement absent des débats pour terminer par une étude partielle du rôle controversé qu'y joua le pays, en Pologne, la victimisation totale généralement admise fait lentement place à la question de la culpabilité (question qui finit par être soulevée en Slovaquie également). En Pologne, la controverse de 
Jedwabne illustre l'énorme impact que peuvent avoir les facteurs externes (cf. la publication du livre de Gross).

Cependant, ce processus d'intégration connut également des rechutes et des revers importants dans les deux pays. La frénésie avec laquelle certaines phases du débat étaient menées (même lorsque thématiquement on pouvait faire état d'une certaine ouverture du débat), et le manque d'enthousiasme lorsqu'il s'agissait de résoudre la question de la culpabilité (par exemple l'attitude de négation détournée qui caractérisa certaines étapes du débat dans les deux pays), en sont de bons exemples. En ce qui concerne cette dernière caractéristique, nous ne considérons pas que le comportement des deux pays au cours de la Deuxième Guerre mondiale (collaboration et résistance massive) ait eu un impact décisif sur la reconnaissance de leur culpabilité. Ce facteur pourrait en partie être tenu responsable du fait que le sujet n'ait pas encore été évoqué en Slovaquie de la même façon qu'en Pologne (l'affaire Jedwabne), mais il se pourrait tout aussi bien que ce ne soit qu'une question de temps ou un manque d'encouragement de l'extérieur. En outre, comme nous l'avons vu, l'histoire de la coexistence avec les Juifs comporte des pages noires dans les deux pays et il faudra du temps pour pouvoir les tourner.

\section{NOTES}

1. Parmi les publications récentes, citons par exemple :Snyder T., "Holocaust: The Ignored Reality " (Holocauste : la réalité ignorée), in : «THE NEW YORK REVIEW OF BOOKS », vol. 56, n 12, 16 juillet 2009 (http://www.nybooks.com/articles/22875) et The Shoah in Ukraine. History, Testimony, Memorialization (La Shoah en Ukraine. Histoire, témoignage et Mémorialisation)(Sous la direction de Brandon R. et Lower W.), Indiana University Press, 2008, 392 p. Parmi les films grand public récents : Der Untergang (La Chute, 2005), realisé par O. Hirschbiegel, The Boy in the Striped Pyjamas (Le Garçon au pyjama rayé, 2008), réalisé par M. Herman et The Reader (Le Liseur, 2008), realisé par St. Daldry.

2. Là aussi le débat ne put commencer avant les années 1960 en raison du caractère délicat du sujet.

3. Le nombre de Juifs polonais et slovaques tués pendant la Deuxième Guerre mondiale provient de : Davies N., God's Playground. A History of Poland (La Plaine de jeu de Dieu. Une histoire de Pologne), vol. II, Columbia University press, New York, 1982, 463 p.; Kamenec I., On the trail of tragedy (Sur les traces de la tragédie), H\&H, Bratislava, 2007, p. 248 (titre original : Po stopach tragédie, Archa, Bratislava, 1991) et Landau R. S., The Nazi Holocaust. Its History and Meaning (L'Holocauste nazi. Son histoire et sa signification), I. B. Tauris, London/New York, 2006, 316 p.

4. Ce terme allemand est souvent employé dans la littérature érudite pour désigner le moyen de gérer certaines périodes de l'histoire ou événements du passé.

5. Sur l'histoire, la mémoire et l'oubli, voir : Ricoeur P., La mémoire, l'histoire, l'oubli, Paris, éd. du Seuil, 2003, 689 p.; les nombreuses publications d'Aleida et Jan Assmann (par exemple: Assmann J., Der lange Schatten der Vergangenheit. Erinnerungskultur und Geschichtspolitik [La Longue Ombre du passé. La Culture du souvenir et la politique de l'histoire), C. H. Beck, Munich, 2006, 320 p.]. Très utile également : Berger St., «Introduction », in : Narrating the Nation: Representations in History, Media and the Arts (Narrer la nation: Ses représentations dans l'histoire, les médias et 
les arts) (Sous la direction de Berger St., Eriksonas L. \& Mycock A.), Berghahn Books, New York/ Oxford, 2008, pp. 1-16.

6. En l'espace de six ans, les pertes dans la population de l'ancienne République de Pologne s'élevèrent à 6028000 . Outre le grand nombre de victimes du côté polonais, une écrasante majorité de meurtres en général eut lieu sur le territoire polonais: sur une estimation de 18 millions de victimes toutes nationalités confondues, plus de 11 millions périrent sur le territoire polonais occupé. Voir Davies N., Op. cit., p. 463.

7. Cf. par exemple les pogroms dirigés contre les Juifs (notamment celui de Jedwabne [voir plus loin]) qui se sont déroulés en Pologne après la conquête allemande des régions de l'est du pays (fin juin 1941) auparavant occupées par les soviétiques.

8. Le 9 septembre 1941, le Parlement slovaque autorisa le tristement célèbre Codex juif, qui «devint l'une des lois anti-juives les plus cruelles dans l'histoire moderne d'Europe » (Kamenec I., Op. cit., p. 161). Toutefois, les premières mesures anti-juives dataient déjà de début 1939 (Ibid. ,pp. 53-96).

9. Entre mars et octobre 1942, les deux tiers de la population juive de Slovaquie (soit 57628 personnes) furent déportés en Pologne, officiellement pour y être réimplantés. Quelques centaines d'entre eux seulement survécurent. 24000 Juifs restèrent en Slovaquie pour travailler suite à des mesures exceptionnelles d'ordre économiques, présidentielles ou religieuses ou pour être placés dans des camps ou des centres de travail. La deuxième vague de déportation commença vers la fin de septembre 1944, après la répression du Soulèvement national slovaque et le début de l'occupation allemande en Slovaquie. Environ 13500 Juifs supplémentaires furent déportés, dont 10000 perdirent la vie (Ibid.,p. 248).

10. Cohen S. J., Politics without a past. The absence of history in postcommunist nationalism (Politique sans passé. L'absence d'histoire dans le nationalisme postcommuniste), Duke University Press, Durham, 1999, p. 8.

11. Narrating the Nation, Op. cit., p. 14. Pour découvrir l'une des quelques études récentes sur le rôle de la presse dans la construction des récits nationaux voir : Nationalist Myths and the Modern Media. Cultural Identity in the Age of Globalisation (Les Mythes nationaliste et les médias modernes. L'Identité culturelle à l'ère de la globalisation) (Sous la direction de Brinks J.H., Rock St. \& Timms E.), I. B. Tauris, Londres, 2005, 288 p.

12. Les deux périodiques durent faire face à de sérieux problèmes financiers au cours des années 1990 et changèrent respectivement trois et quatre fois de nom : « NOVÉ SLOVO " (1991-1992), « NOVE SLOVO BEZ REŠPEKTU » (1993-1997), « NOVE SLOVO » (depuis 1998) ; «DOMINO » (1992), « DOMINO EFEKT » (1993-septembre 1996, février 1997-octobre 1997), « DOMINO FORUM » (octobre 1997-2004).

13. «WPROST » et « POLITYKA »sont, qui plus est, les hebdomadaires les plus vendus en Pologne.

14. Pour la Slovaquie, je me base sur l'étude détaillée de l'héritage de la Deuxième Guerre mondiale dans la Slovaquie communiste réalisée par Shari J. Cohen (voir Cohen S. J., Op. cit., pp. 85-117.). Pour la Pologne, je me base sur l'introduction rédigée par Joshua D. Zimmerman ( Contested Memories : Poles and Jews during the Holocaust and its Aftermath [Mémoires contestées : Les Polonais et les Juifs pendant l'Holocauste et ses conséquences] [Sous la direction de Zimmerman J. D.)], Rutgers University Press, New York, pp.1-16), ainsi que sur Stobiecki R., Historiografia PRL. Ani dobra, ani mĄdra, ani piĘkna... ale skomplikowana. Studia $i$ szkice (L'Historiographie de la République Populaire de Pologne. Ni bonne, ni intelligente, ni belle... mais compliquée. Étude et esquisse), Wydawnictwo TRIO, Varsovie, 2007, 361 p.

15. Voir introduction.

16. Jusqu'alors, les historiens slovaques étaient une denrée rare et l'histoire nationale n'avait été que très peu écrite. Au cours des années qui suivirent, le processus d'écriture se développa à l'Institut historique, qui fut fondé à l'Académie des Sciences en 1950, ainsi qu'à l'Institut d'histoire du Parti communiste. 
17. Ceci en opposition totale avec l'image négative perçue à l'étranger du comportement polonais pendant la guerre : par exemple en Israël, voir Contested Memories, Op. cit., p. 1.

18. Le premier de ces travaux, rédigé par Terra Berenstein et Adam Rutkowski de l'Institut historique juif de Varsovie, fut publié en 1963. D'autres exemples de ce type de publications: Bartoszewski W. \& Lewinówna Z., Ten jest $z$ ojczyzny mojej (Il est de ma patrie), 2 vol., Znak, Cracovie, 1966 \& 1969, 928 p.; Bartoszewski W. \& Lewin S., Righteous among Nations: How Poles Helped the Jews. 1939-1945 (Les Justes parmi les Nations : Comment les Polonais ont aidé les Juifs), Earlscourt Publications, Londres, 1969, 834 p. ; Bartoszewski W. \& Lewin S., The Samaritans: Heroes of the Holocaust (Les Samaritains: Héros de l'Holocauste), Twayne Publisher, New York, 1970, 442 p. ; Datner S., Las sprawiedliwych (La Forêt des justes), KsiĄżka i Wiedza, Varsovie, 1968, 115 p. ; Iranek-Osmiecki K., Kto ratuje jedno życie - Polacy i Żydzi. 1939-1945 (Qui sauve une vie Polonais et Juifs), Orbis, Londres, 1968, 391 p. (publié en anglais sous le titre He Who Saves One Life, Crown Publishers, New York, 1971, 336 p.).

19. Ce qui impliqua des conférences internationales, l'apparition de journaux spécialisés consacrés aux analyses judéo-polonaises, et la fondation de quatre nouveaux centres de recherches consacrés aux études judéo-polonaises (Cf. Contested Memories..., Op. cit., pp. 4-5).

20. Les travaux de Teresa Prekerowa, Nechama Tec et Alina Cała furent particulièrement importants.

21. Błoński J., « Biedni Polacy patrzą na getto » (Les pauvres Polonais regardent le ghetto), in : « TYGODNIK POWSZECHNY ", $\mathrm{n}^{\circ} 2,1987, \mathrm{p} .1$.

22. Stachura P. D., Poland. 1918-1945: An Interpretive and Documentary History of the Second Republic (Pologne. 1918-1945: Une histoire interprétative et documentée de la Seconde République), Routledge, Londres/New York, 2004, p. 146.

23. Dans les manuels utilisés à cette époque qui ont été analysé par Cohen, les Juifs slovaques sont complètement absents.

24. Il y eut tout d'abord deux œuvres de fiction largement distribuées: la nouvelle de Rudolf Jašik, Námestie Svatej Alžbety (La Place Sainte-Elisabeth, 1959) et le film Shop on Mainstreet de J. Kadár \& E. Klos (1965, interdit après 1968). D'autres œuvres fictionnelles et non fictionnelles : Lipták Lubomír, Slovensko v 20. storočie (La Slovaquie au XX ${ }^{\mathrm{e}}$ siècle, 1968, interdit après 1969) et le travail d'Ivan Kamenec qui ne sera finalement publié qu'en 1991.

25. Cohen S. J., Op. cit., p. 119.

26. La Tchécoslovaquie devint une fédération avec l'adoption de la Loi constitutionnelle de la fédération tchécoslovaque du 27 octobre 1968.

27. Le roman de Milan Kundera, Kniha smíchu a zapomnění (Le Livre du rire et de l'oubli, 1979), aborde le sujet de l'oubli au cours de cette période.

28. Les seules exceptions étaient les textes d'histoire de l'université et de la faculté de droit qui fournissaient plus d'informations sur le chapitre juif (par exemple des détails sur les lois raciales, sur l'aryanisation et sur les déportations : Cohen S. J., Op. cit., p. 113).

29. Contrairement à ce que certains journaux (étrangers) affirmèrent, Lech WałĘsa ne s'excusa absolument pas pour l'Holocauste. Il reconnut en revanche que les Polonais (aussi) avaient commis des crimes contre les Juifs («Il y a eu, parmi nous, des criminels»). Malgré quelques réactions négatives dans les cercles nationalistes extrémistes en Pologne, le geste de Wał‡̨sa fut généralement bien accueilli à l'étranger et dans son propre pays.

30. Du côté slovaque, de gros progrès avaient déjà été faits avant novembre 1993 (lorsque le Parlement slovaque avait adopté une loi pour la restitution des biens juifs à caractère religieux, avec un effet rétroactif remontant à novembre 1938, date à laquelle les premières mesures antijuives avaient été mises en application), mais du côté polonais il fallut attendre jusqu'en 1997 pour voir la première tentative de législation. Qui plus est, cette loi n'était applicable que pour les Juifs qui vivaient toujours en Pologne. Pour en savoir plus sur la couverture de ce sujet par la presse slovaque et polonaise, voir Lenická A., « Komu prospeje hlboká orba do reštitúcií? » (À qui 
profite le vacarme autour des restitutions), in : «SLOvo», n³,1992, p. 3 ; Wróblewski A. K., «Zwrot majątku Żydom. Nasze ulice, wasze kamienice» (La Restitution des biens aux Juifs. Nos rues, vos maisons), in : «POLITYKA », nº 17 (27/4), 1996, p. 31 ; Ostrowski M., «Spór o zwrot » (La Controverse sur la restitution), in : « POLITYKA », $n^{\circ} 4,1997$, p. 13.

31. C'est à peu près à cette époque que l'on commença à travailler sur la construction du Mémorial de l'Holocauste à Bratislava, situé près de la Cathédrale St-Martin, à l'emplacement de l'ancienne synagogue. Pour en savoir plus sur les monuments postcommunistes dédiés aux Juifs dans la capitale polonaise, voir Van Cant K., «Historical memory in post-communist Poland: Warsaw's monuments after 1989 ", in: "STUDIES IN SLAVIC CULTURES", n 8, University of Pittsburgh, 2009, pp.90-118. La publication slovaque la plus conséquente sur l'Holocauste du début des années 1990 est Kamenec I., Op. cit. Un exemple de film sorti à cette époque : Europa, Europa (1991) par la réalisatrice polonaise Agnieszka Holland.

32. Lenická A., Art.op., p. 3 ; Babic M., «Radoví Nemci a holocaust. Americký historik Daniel Goldhagen o Hitlerových ochotných popravcoch " (Les Hommes de troupe allemands et l'holocauste. L'historien américain Daniel Goldhagen au sujet des exécuteurs hitlériens dociles), in : « NOVE SLOVO BEZ REŠPEKTU », n 34, 1996, p. 14.

33. La question de savoir si l'identité nationale slovaque était forte en 1993, et, si oui, à quel point elle l'était, est sujet à discussion, mais n'est pas utile pour mon propos. Ce qui est certain en revanche, c'est que la création d'un nouvel état implique naturellement un processus de construction de la nation, peu importe que l'identité/la conscience nationale du peuple soit forte ou non.

34. En théorie, nous devrions également mentionner la République soviétique de Slovaquie de 1919, un état communiste qui ne resta pas longtemps en place (du 16 juin au 7 juillet) dans le sud et l'est de la Slovaquie actuelle. Cependant, comme cette république n'a pas existé pendant plus d'un mois et était issue du bolchevisme, on ne lui reconnaît généralement pas le statut d'État slovaque indépendant

35. Cohen S. J., Op. cit., pp. 123-132.

36. Par exemple : Beňa J., "Slovenská národná štátnoš̌ a roky 1939-1945 » (L'Etat national slovaque et les années 1939-1945), in : « NOVE SLOVO BEZ REŠPEKTU », nº 27, 1994, pp. 27-29.

37. Idem.

38. Quelques exemples: Kulíková J., «V záujme svedomia » (Dans l'intérêt de la conscience), in : «SLovo ", $\mathrm{n}^{\circ} 10,1994$, p. 10 ; sn., «Spomienka na November » (Souvenir de novembre), in : « NOVE SLOVO BEZ REŠPEKTU», n 17, 1995, p. 13; sn, « Heslo dňa - hysterizácia » (Mot du jour : hystérisation), in : « NOVE SLOVO BEZ REŠPEKTU », n² 20, 1996, p. 12 ; Poleda, st, in : « NOVE SLOVO BEZ REŠPEKTU », n 17, 1997, p. 6 ; Štrasser J., «Infantilný predpenzista. Malý sprievodca ihravým Slovenskom» (Le Prépensionné infantile. Petit guide de la Slovaquie joueuse), in: «DOMINo EFFEKT ", n 48, 1995, p. 3 ; Smolec J., st, in : « DOMINO EFEKT », n 17, 1996, p. 8 ; Bútora M., " Slová a činy vtedy a dnes » (Les Paroles et les actes, hier et aujourd'hui), in : « DOMINO FORUM », 1997, p. 2.

39. Cohen S. J., Op. cit., p. 150.

40. Abréviation de «Strana demokratickej L’avice » (Parti démocrate de gauche).

41. La campagne pour l'introduction du 29 août comme jour férié en Slovaquie, largement soutenue par le SDL, en est un bon exemple (Cf. Krno M., «O rok ako štátný sviatok? » [Et dans un an, fête nationale ?], in : «sLovo ", $\mathrm{n}^{\circ}$ 1, 1992, p. 1).

42. Cette tendance, que l'on retrouve également chez une partie des jeunes historiens slovaques d'aujourd'hui, fut sévèrement critiquée par les historiens slovaques (voir par ex. Kamenec I., Op. cit., p. 15). Selon ces derniers, il est impossible de séparer l'état de son régime dictatorial (et antisémite).

43. sn, «W Oświęcimiu » (À Auschwitz), in : «POLITYKA», n² 29, 1989, p. 2 ; Fandrejewska A., «Konflikt w OświĘcimiu » (Conflit à Auschwitz), in:«POLITYKA», n 30, 1989, p.11; 
Dombrowski D., « Karmelitanki w OświĘcimiu » (Les Carmélites à Auschwitz), in : « POLITYKA », n - 31, 1989, p. 2 ; Poklewski-Koziełł K., « Spór o klasztor w Ośwį̨cimiu - w oczach prawnika » (La Controverse du couvent à Auschwitz à travers le regard d'un juriste), in : « POLITYKA », n 36, 1989, p. 4 ; Gebert K., « TrochĘ mniej w domu » (Un peu moins à la maison), in : « POLITYKA », n 36, 1989, p. 10 ; Ławniczak A., « Krzyż na górze Synaj » (Une croix sur le Mont du Sinaï), in : «WPRosT », n - 1, 1990, pp. 20-22.

44. Pour un compte-rendu détaillé sur la controverse du Carmel, voir Bartoszewski W., The Convent at Auschwitz (Le Couvent à Auschwitz), George Braziller, New York, 1991, 161 p.

45. sn, st, in : « POLITYKA », $n^{\circ} 17,1993$, p. 2.

46. Entre-temps, la discussion s'était écartée du Carmel pour se pencher sur l'affaire de la Croix catholique d'Auschwitz, plantée près du couvent. Cf. : Glemp J., st, in : «WPROST ", n 14, 1998, p. 10 ; WałĘsa L., "Ogórki, krzyże i wybory » (Les Cornichons, les croix et les élections), in: « WPROST ", n 35, 1998, p. 32.

47. Par exemple : Grynberg H., « Rachunki krzywd » (Le Prix des préjudices), in : «WPROST », n²5, 1994, p. 60.

48. Mac J. S., « Sprawiedliwość sprawiedliwym » (La Justice aux justes), in : « WPRosT », n 49, 1994, pp. 29-30 ; Turski M., « Docenić bohaterstwo ", in : POLITYKA, n²14, 1994, p. 31.

49. Sawka H., st, in : «WPROST », $\mathrm{n}^{\circ} 28,1995$, p. 15.

50. C'est-à-dire qu'il ne constituait jamais le sujet principal d'un article, mais figurait souvent au second plan d'une publication liée aux Juifs.

51. Les critiques lancées contre la campagne antisémite de 1968 sont de bons exemples. (Andrzejewski P., «Droga » [Le Chemin], in : « WPRosT », $\mathrm{n}^{\circ} 11,1990$, p. 3) ; pour des exemples de plaisanteries sur l'antisémitisme et la xénophobie en général des Polonais, voir Sawka H., st, in : "WPROST ", $n^{\circ} 5,1992$, p. 7) ; pour un article sur les stéréotypes employés par les Polonais contre les Juifs, et sur les relations controversées entre Juifs et Polonais pendant l'entre-deux guerre et la Deuxième Guerre mondiale, voir KTT, «Żyd poczciwy OjczyznĘ... » (Le Juif honnête face à la patrie...), in : « POLITYKA $, \mathrm{n}^{\circ} 45,1993, \mathrm{p} .28$.

52. Les articles sur l'Holocauste publiés à l'occasion des anniversaires, et qui portent sur les désaccords entre Juifs et Polonais en guise de commémoration, constituent de bons exemples : Graczyk M. (rozmowa z Maurice'em Goldsteinem [conversation avec Maurice Goldstein]), «Większa mniejszość» (La plus grande minorité), in: «WPROST», n 5, 1995, pp. 21-22; Graczyk M., Cywiński P. \& Piasecki W., «Rachunek sumień» (Le Prix des consciences), in: «WPROST ", n 7, 1995, p. 43.

53. Les articles suivant illustrent bien le premier temps décrit dans ce paragraphe: Dian A., «Politická emigrácia a samostatnoš̌ Slovenska I.» (La Politique de l'émigration et l'indépendance de la Slovaquie), in : « NOVE SLOvo BEZ REŠPEKTU », n 17, 1997, pp. 32-33 ; Greguš P., « Mýty a pravdy o 14. marci 1939 » (Mythes et réalités autour du 14 mars 1939), in : « SLovo ", n - 3, 1999 (http://www.noveslovo.sk/archiv/1999-03/str11.html).

54. Bunea A., « Remembering the Holocaust. An Inquiry into the Fascinating and Intricate World of Holocaust Memory" (Se souvenir de l'Holocauste. Enquête dans le monde complexe et fascinant de la mémoire de l'Holocauste), in : « STUDIA HEBRAICA », n 5, 2005, p. 256.

55. Pour un aperçu du débat de presse couvrant ces événements, voir Kersten K., « RĘka Polaka. Pogrom kielecki : nadal więcej pytań niż odpowiedzi» (La Main du Polonais. Le progrom de Kielce: encore plus de questions que de réponses), in: « POLITYKA», n²3, 1996, pp.68-71; Grochowska M., « Rysa na krysztale. Kielce, 50 lat później » (Une fissure dans le cristal. Kielce, 50 ans après », in : « POLITYKA ", n² 28, 1996, p. 16 ; Kersten K., "Sprawa pogromu kieleckiego (L'Affaire du pogrom de Kielce), in : « POLITYKA», n 43 (25/10), 1997, p. 12 ; Nowak St. (courrier des lecteurs), «Psychoza mordu » (La Psychose du meutre), in : « POLITYKA », n 46, 1997, p. 98. 
56. Après une nouvelle analyse des faits et des circonstances entourant le pogrom de Kielce (à l'initiative de la Commission principale d'enquête sur les crimes commis en Pologne/ Główna Komisja Badania Zbrodni przeciw Narodowi Polskiemu), il n'y a toujours pas de réponse univoque à la question visant à déterminer si le pogrom a été volontairement provoqué par les autorités communistes ou pas. Ce qui est certain, c'est que même si le pogrom s'avérait être un cas de provocation, il résulterait toujours de préjugés antisémites, de phobie et de rancœurs de la société polonaise.

57. Gross J. T., SĄsiedzi. Historia zagłady żydowskiego miasteczka, Fundacja Pogranicze, Sejny, 2000, 157 p.Édition Française : Jan T. Gross, Les Voisins. 10 juillet 1941, un massacre de Juifs en Pologne, Fayard, 2002, 285 p.

58. Błoński J., Art. cit., p. 1.

59. Dans son article «Collective Remembrance in Jedwabne. Unsettled Memory of World War II in Postcommunist Poland» (Souvenir collectif à Jedwabne. Mémoire perturbée de la Deuxième Guerre mondiale dans la Pologne postcommuniste) (in: " HISTORY \& MEMORY ", n 18, 2006, pp.152-178), Ewa Wolentarska-Ochman analyse les effets néfastes de cette approche critique adoptée par la presse polonaise sur le travail de mémoire entrepris dans la ville de Jedwabne.

60. Les représentants les plus importants de ce genre de presse étaient : « GŁOS », « NASZA POLSKA », « NASZ DZIENNIK », « MYŚL POLSKA ».

61. Bunea A., Art. cit., p. 256.

62. Kurczewski J., «Mord rytualny » (Meurtre rituel), in: «WPROST», n 50, 2000 (http:// www.wprost.pl/ar/8696/Mord-rytualny/?I=941).

63. Idem

64. Cf. Nowina-Konopka P., «Wina bezwstydnych» (La Faute des impudents), in : «WPROST », n -11, 2001 (http://www.wprost.pl/ar/9536/Wina-bezwstydnych/?I=955) ; Nowak Jeziorański J., « Dossier », in : «WPROST », n 11, 2001 (http://www.wprost.pl/ar/9544/Dossier/?I=955).

65. Kaczyński A., «Całopalenie » (L'Holocauste), in : «RZECZPosPolitA », 5 mai 2000 (http://newarch.rp.pl/artykul/274868_Calopalenie.html).

66. Kurczewski J., Art.cit.

67. Zieleniewski M., «BrakujĄce ogniwo" (Le Maillon manquant), in: «WPRosT ", n 4, 2001 (http://www.wprost.pl/ar/9106/Brakujace-ogniwo/?I=948); Mac J. S., Borowski J., «Test z Jedwabnego » (Test de Jedwabne), in : «WPROST », n 11, 2001 (http://www.wprost.pl/ar/9512/ Test-z-Jedwabnego/?I=955) ; Warszawski D., «Dwie Polski w Jedwabnem » (Deux Polonaises à

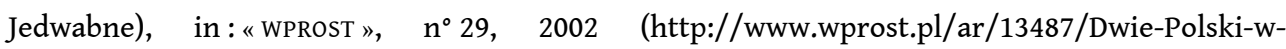
Jedwabnem/?I=1025).

68. Wokół Jedwabnego (Autour de Jedwabne) (Sous la direction de Machcewicz P. \& Persak K.), IPN, Varsovie, 2002, vol. 1 : Studia (Étude), 525 p., \& vol. 2 : Dokumenty (Documents), 1034 p.

69. Cf. Kurczewski J., Artcit.; sn, «Jedwabieński pomnik» (Le Monument de Jedwabne), in: "POLITYKA ", n 12, 2001, p. 12.

70. Dans son article «The Catholic Church in Poland under Nazi Occupation (1939-1945) and the first Years of Communism (1944-1948) » (L'Église catholique en Pologne sous l'Occupation nazie [1939-1945] et les premières années du communisme [1944-1948]) (in : Religion under siege [La Religion en état de siège] [Sous la direction de BankJ. \& Gevers L.), Annua Nuntia Lovaniensia, Louvain,2007, pp. 1-38), Idesbald Goddeeris suggère que l'absence du pogrom de Radziłów du récit historique officiel polonais est liée aux répercussions qu'il pourrait avoir sur l'image de l'Église catholique de Pologne (des sources pointent du doigt l'indifférence du curé de la ville, Aleksander Dogolewski, qui refusa d'apporter son aide aux habitants juifs de Radziłów).

71. Weiss S., «Braterstwo pamięci » (La Fraternité de la mémoire), in : «WPROST », n 30, 2004 (http://www.wprost.pl/ar/63476/Braterstwo-pamieci/?I=1130). 
72. Des passages tirés des mémoires d'une victime juive pour être publiés constituent de bons exemples de ceci : Hrabovecká H., « Rozhovor s vnučkou. Každá vláda ma v práve pokračovat “a bezprávie naprávat" » (Conversation avec ma petite-fille. Chaque gouvernement doit persévérer dans le droit et "réparer l'injustice"), in: «SLOvo», n 11, 2001 (http://www.noveslovo.sk/ archiv/2001-11/ominulosti.html\#01).

73. Samson I., « ... Berlín alebo Vatikán?... » (... Berlin ou le Vatican ?...), in : « DOMINo ForUM », n 12, 1998, pp. 7-9.

74. L'une des raisons de cette attitude tenait au flou dans l'historiographie slovaque qui continuait d'entourer les indulgences présidentielles que Jozef Tiso aurait attribuées à un certain nombre de Juifs. Depuis lors, James Mace Ward est parvenu à combler ce vide en apportant des données sur le nombre d'exemptions et sur les circonstances dans lesquelles elles avaient été attribuées. Cf. Mace Ward J., " "People Who Deserve it": Jozef Tiso and the presidential exemption» (Les Gens qui le méritent: Jozef Tiso et l'exemption présidentielle), in «NATIONALITIES PAPERS », vol. 30, n 4, 2002, pp. 571-601.

75. Vagovič M. (dans une interview avec l'historien Kamenec), « Tiso bol Hitlerom fascinovaný » (Tiso était fasciné par Hitler), in : «SLovo »,n³7, 2000, pp. 4-5; Hradská K., "Smutné výročie » (Un triste jubilé), in : « DOMINO », n 36, 2001, p. 8.

76. Exemple : Samson I., Art. cit.

77. Exemples : Durániková J., « Peklo menom Sobibor » (Un enfer nommé Sobibor), in : «DOMINO » ,n 14, 2002, p. 8 ; Ďurková K., « V továrni smrti » (Dans la fabrique de la mort), in : «DOMINO FORUM », $\mathrm{n}^{\circ}$ 21, 2002, p. 9 ; Durániková J., « Majdanek cintorín Európy » (Majdanek, cimetière de l'Europe), in : «DOMINO FORUM», n 35, 2002, p. 16 ; un autre exemple de la présence de l'horreur nazie dans le discours de la presse slovaque de l'époque: Duránikováj., «Hartheim. Škola masového vraždenia » (Hartheim. L'École du massacre de masse), in : «DOMINO FORUM », n 42, 2002, p. 9.

\section{AUTEURS}

\section{KATRIN VAN CANT}

Doctorante à l'Université Catholique de Leuven, aspirante FWO 